\title{
Article
}

\section{Gold-conjugated nanobodies for targeted imaging using high- resolution secondary ion mass spectrometry}

\author{
Paola Agüi-Gonzalez ${ }^{1,2}$, Tal M. Dankovich ${ }^{2}$, Felipe Opazo ${ }^{1,2,3}$, Silvio O. Rizzoli ${ }^{1,2 *}$, and Nhu T. N. Phan ${ }^{1,2,4 *}$ \\ 1 Center for Biostructural Imaging of Neurodegeneration, University Medical Center Göttingen, von- \\ Siebold-Straße 3a, 37075 Göttingen (Germany) \\ 2 Department of Neuro- and Sensory Physiology, University Medical Center Göttingen, Humboldtallee 23, \\ 37073 Göttingen (Germany) \\ 3 NanoTag Biotechnologies GmbH, Rudolf Wissell-Str. 28a, 37079 Göttingen (Germany) \\ 4 Department of Chemistry and Molecular Biology, University of Gothenburg, Kemivägen 10, 41296, \\ Gothenburg (Sweden) \\ * Correspondence: thi.phan@med.uni-goettingen.de (nhu.phan@chem.gu.se)
}

\begin{abstract}
Nanoscale imaging with the ability to identify cellular organelles and protein complexes has been a highly challenging subject in secondary ion mass spectrometry (SIMS) of biological samples. This is because only a few isotopic tags can be used successfully to target specific proteins or organelles. To address this, we have generated gold-nanoprobes, in which gold nanoparticles are conjugated to nanobodies. The nanoprobes were well suited for specific molecular imaging using NanoSIMS at subcellular resolution. They demonstrated to be highly selective to different proteins of interest, and sufficiently sensitive for SIMS detection. The nanoprobes offer the possibility of correlating the investigation of cellular isotopic turnover to the positions of specific proteins and organelles, thereby enabling an understanding of functional and structural relations that are currently obscure.
\end{abstract}

Keywords: nanobodies; gold nanoparticles, target imaging; nanoSIMS; SIMS

\section{Introduction}

Secondary ion mass spectrometry (SIMS), an imaging technique featured with high spatial resolution, molecular specificity, and high sensitivity, has become a valuable analytical tool for molecular imaging in biological research. The technique enables the analysis of multiple analytes in a single measurement at subcellular resolution providing a better understanding of a complex interaction of cellular molecules and structures. NanoSIMS particularly allows the detection of elemental and small ion species at the spatial resolution of $50 \mathrm{~nm}$, which is comparable to various super-resolution light microscopy and electron microscopy techniques. However, SIMS exhibits a drawback: It is difficult to identify cellular structures and localize specific proteins, which are necessary for an insight into the relationship between molecular organization and function at a subcellular level.

A common approach to complement the information of morphology or protein localization is to correlate SIMS with other imaging techniques such as electron microscopy, fluorescence microscopy, or matrix-assisted laser desorption ionization (MALDI) [1-3]. Correlative imaging offers the means to connect different properties, structures and functions of the cells. However, it is highly challenging due to the compatibility requirements of the instrumentation and sample preparation. An alternative approach is to employ probes to label specific proteins which are then detectable by SIMS. This is a more straightforward solution alleviating the complication of the correlation method. Target molecular imaging with NanoSIMS and SIMS has been 
a long-searched topic in the field. Up to now, labeling probes for specific proteins have been available to a very limited extent due to their highly challenging development process. A suitable probe must satisfy several requirements. It should contain elements that are easily ionized and rarely present in biological specimens, and it selectively binds to the target molecules with sufficient density to be detectable in SIMS. A few labeling probes were recently developed and successfully applied to image different proteins in cells at nanoscale resolution, for example, dual probes containing isotopic element boron, or fluorine, and a fluorophore for SIMS and fluorescence microscopy imaging [4-6]. Such small-sized probes are beneficial to SIMS imaging regarding the imaging resolution, labeling precision and labeling density. However, they often exhibit difficulty detecting low abundant molecules due to a high background signal level, which comes from the embedding resin and the substrate materials commonly used for NanoSIMS and SIMS experiments, particularly silicon wafers.

Labeling probes containing metal coupled to antibodies that can be detected by NanoSIMS and EM have been employed for imaging biological samples ${ }^{[7-9]}$. For example, colloidal gold coupled antibodies were used to image actin and synaptophysin in mammalian cells ${ }^{[10]}$. In this case, the tissue was fixed, embedded in LR White plastic resin, cut into sections that were subsequently immunostained with a colloidal gold coupled antibody. This post-embedding staining approach often encounters an issue that many epitopes in the sample are not revealed due to the limited ability of the antibody to penetrate into the resin-embedded sections. In addition, the chemical fixation and tissue preparation before the embedding are often not optimal for antigen preservation, which results in a low detected signal of the molecules of interest. To increase the epitope accessibility in the embedded samples, optimizations for the conditions of resin polymerization or a modification of the resin embedded samples, such as resin etching with Na-ethanolate and antigen retrieval by sodium dodecyl sulfate at $0.5 \%$ were reported [11,12].

Lanthanides were coupled to antibodies for detecting multiple proteins in human breast cancer tissues using NanoSIMS [13]. Nevertheless, the large size of the antibodies could be a limiting factor for the spatial resolution of NanoSIMS imaging due to the possible clustering of the antibodies. The clustering also restricts the accessibility of the probes to all the epitopes that could hinder the detectability of the molecules [14]. Lanthanides and gold have been commonly used for imaging in biological and medical studies due to their bio-compatibility ${ }^{[15-17]}$. Particularly, gold nanomaterials have been extensively used in mass spectrometry imaging because of their well-established synthetic procedures which enable fully customized gold nanomaterials ${ }^{[15]}$. For SIMS imaging, the secondary ion yield heavily depends on the ionization energy for positive ions and the electron affinity for negative ions. Due to their low ionization energy ${ }^{[18]}$, lanthanides are easily ionized and thus are more suitable for detection in positive ion mode ${ }^{[13]}$. On the other hand, gold is one of the six elements having the highest electron affinity (only after chlorine, fluorine, bromine, iodine, and astatine $)^{[19]}$, therefore it is well suited for detection in negative ion mode.

In this study, we tested nanoprobes consisting of gold nanoparticles (Au NPs) conjugated to specific nanobodies to reveal specific proteins using nanoSIMS imaging. The nanobodies are very small in size $(\sim 3 \mathrm{~nm}$ in length) and thus are more compatible with NanoSIMS imaging at nanoscale resolution. In addition, it helps to reduce the risk of clustering and epitope inaccessibility possibly caused by the antibodies. Two types of nanoprobes were used. The first one consists of a nanobody that can bind directly and specifically to endogenous proteins like the vesicular glutamate transporter 1 (Au antivGlut1 nanobody). The second type of Au nanoprobe makes use of a nanobody that can bind specifically to mouse immunoglobulins ( $\mathrm{Au}$ anti-mouse secondary nanobody). We employed these nanoprobes to label different proteins in primary cultures of rat hippocampal neurons to demonstrate their potential for target bio-imaging with NanoSIMS.

\section{Materials and Methods}




\subsection{Coupling procedure for Au NPs-anti-mouse secondary nanobody}

The reactive anti-mouse nanobody bearing 1 ectopic cysteine (N1202; NanoTag Biotechnologies) was used for the coupling reaction with $3 \mathrm{~nm}$ mono-maleimide Au NPs (C11-3 MMAl-DRY-2.5; Nanopartz Inc., USA). The coupling was performed similarly as previously described ${ }^{[4,5]}$. In brief, $\sim 30 \mathrm{nmol}$ of the nanobody containing C-terminal extra cysteine was reduced using $10 \mathrm{mM}$ Tris(2-carboxyethyl)phosphine hydrochloride (TCEP) in PBS at $\mathrm{pH} \sim 7$ for 1 hour on ice. Excess of TCEP was then removed using a NAP5 column (GE Healthcare) equilibrated with ice-cold and degassed PBS at $\mathrm{pH} \sim 7$. The reduced nanobody was mix immediately with $\sim 50 \mathrm{nmol}$ of thiol reactive Au NPs. Reaction was left for $2 \mathrm{~h}$ on ice. Coupled nanobodies were purified using an Äkta HPLC system and a size-exclusion column (Superdex 75 increase, 10/300 GL). A chromatograph of the purified $\mathrm{Au}$-anti-mouse nanobody conjugation is showed in Figure S1.

The anti-vGlut1 nanobody (N1602, NanoTag Biotechnologies) conjugated to monomaleimide $1.4 \mathrm{~nm}$ colloidal gold from Nanoprobes, Inc., was purchased as a custom product from NanoTag Biotechnologies $\mathrm{GmbH}$.

\subsection{Cell culture and preparation}

Hippocampal neurons isolated from rat brains (P0) were seeded on silicon wafers and cultured in $\mathrm{N} 2$ medium at $37^{\circ} \mathrm{C}$ in a humid atmosphere with $\mathrm{CO}_{2} 5 \%$ for two weeks. To prepare for experiments, neuronal cells were fixed with paraformaldehyde (PFA) $4 \%$ in PBS for $30 \mathrm{~min}$ at RT, quenched with glycine $100 \mathrm{mM}$ in PBS, permeabilized, and blocked with a permeabilizing/blocking solution containing BSA $2.5 \%$ and Triton X-100 $0.1 \%$ in PBS for $1 \mathrm{~h}$ at RT.

Direct immunostaining

After being fixed, quenched, blocked, and permeabilized, the cells on the silicon wafers were incubated with the $\mathrm{Au}$ anti-vGlut1 nanobody in the permeabilizing/blocking solution at RT for $1 \mathrm{~h}$ followed by washing with BSA $2.5 \%$ in PBS for $3 \times 5 \mathrm{~min}$. The labeled cells were then washed sequentially with PBS, high salt PBS ( $\mathrm{NaCl} 362 \mathrm{mM}$ in PBS, pH 7.4), PBS, and MQ water, followed by air-drying before NanoSIMS imaging.

Indirect immunostaining

After being fixed, quenched, blocked, and permeabilized, the cells on the silicon wafers were incubated with the mouse antibody of the protein of interest (POI), particularly a synaptic protein Synaptotagmin 1 (SySy, 105311) or a mitochondrial marker TOM20 (Sigma, WH0009804M1), in the permeabilizing/blocking solution at RT for $1 \mathrm{~h}$ followed by washing with BSA $2.5 \%$ in PBS for $3 \times 5$ min. Afterward, cells were incubated with the $\mathrm{Au}$ anti-mouse secondary nanobody in the permeabilizing/blocking solution at RT for $1 \mathrm{~h}$ followed by washing with BSA $2.5 \%$ in PBS for $3 \times 5 \mathrm{~min}$. The labeled cells were then washed sequentially with PBS, high salt PBS ( $\mathrm{NaCl} 362 \mathrm{mM}$ in PBS, pH 7.4), PBS, and MQ water followed by air-drying before NanoSIMS imaging.

\subsection{Confocal microscopic imaging}

For the confocal imaging experiments, hippocampal neurons were plated on glass coverslips. The neurons were fixed, and immunostained against TOM20 or vGlut1 (STAR635P-conjugated nanobody; NanoTag N1602), as for the silicon wafers (see Indirect immunostaining section). Following the incubations, the neurons were washed with PBS and then embedded in Mowiol. Confocal imaging was performed on an Abberior QUAD scan STED/confocal microscope (Abberior $\mathrm{GmbH}$, Göttingen, Germany) equipped with a UPlanSApo 100x 1.4 NA objective (Olympus Corporation, Shinjuku, Tokyo, Japan) and an EMCCD iXon Ultra camera (Andor, Belfast, Northern Ireland, UK). The samples were excited using pulsed $485 \mathrm{~nm}, 580 \mathrm{~nm}$, and $640 \mathrm{~nm}$ lasers for imaging the autofluorescence background signal and the proteins of interest vGlut1 and TOM20, respectively. The pinhole size was set to 1 airy unit.

\subsection{NanoSIMS imaging and data analysis}


The NanoSIMS imaging was carried out by a NanoSIMS 50L (Cameca, France) with an $8 \mathrm{kV}$ cesium primary ion source in negative ion mode. A primary ion current of 0.51 pA (D1-3 or D1-4) was used to sputter sample areas of 25-30 $\mu \mathrm{m}$ to obtain images of 256 x 256 pixels or 512 x 512 pixels for imaging vGlut and TOM20. For synaptotagmin, the primary ion current of $\sim 15 \mathrm{pA}$ (D1-1) was used. The sample was first implanted at the primary ion current $\sim 15 \mathrm{pA}$ (D1-1) to obtain a steady state of the sputter rate and ionization before imaging. The analyzed ions were ${ }^{12} \mathrm{C}^{14} \mathrm{~N}-,{ }^{197} \mathrm{Au}^{-}$, and ${ }^{28} \mathrm{Si}_{2}{ }^{-}$, which are expressed as ${ }^{12} \mathrm{C}^{14} \mathrm{~N},{ }^{197} \mathrm{Au}$, and ${ }^{28} \mathrm{Si} 2$ in the paper. NanoSIMS image exportation, drift correction if necessary, stacking from individual image layers, line profile measurement, and image ratio measurement $\left({ }^{197} \mathrm{Au} /{ }^{12} \mathrm{C}^{14} \mathrm{~N}\right)$ were carried out by the NanoSIMS analysis software and the OpenMIMS plugging in ImageJ ${ }^{[20]}$. To compare the ${ }^{197} \mathrm{Au} /{ }^{12} \mathrm{C}^{14} \mathrm{~N}$ ratio signal between samples labeled with Au nanoprobes and negative control samples (labeled with $\mathrm{Au}$ nanoprobe in the absence of primary antibody, and labeled with neither primary antibody nor Au nanoprobe), self-written MATLAB (the MathWorks Inc, Natick, MA) scripts were used. Circular regions of interest (ROI) were manually selected. From each pixel of the ROI, the ${ }^{197} \mathrm{Au} /{ }^{12} \mathrm{C}^{14} \mathrm{~N}$ ratio was extracted and then was averaged across all pixels of the ROI. We then applied a Kolmogorov-Smirnov test to statistically compare the gold signal between the labeled and negative control samples.

\section{Results}

Au NPs with a functional group maleimide were conjugated to the nanobodies having the cysteine residues via the cysteine maleimide reaction. For the direct immunostaining approach, a nanobody against the endogenous protein in the cells was used, as demonstrated here, the Au NPs coupled anti-vGlut1 nanobody (Au anti-vGlut1 nanobody) for labeling the vesicular glutamate transporter 1 . The indirect immunostaining was accommodated with the Au NPs coupled to the nanobody against a light chain of the mouse antibody (Au anti-mouse secondary nanobody) that allows to label any proteins of interest recognized beforehand with a mouse antibody. The labeling strategies using these Au nanoprobes are illustrated in Figure 1.

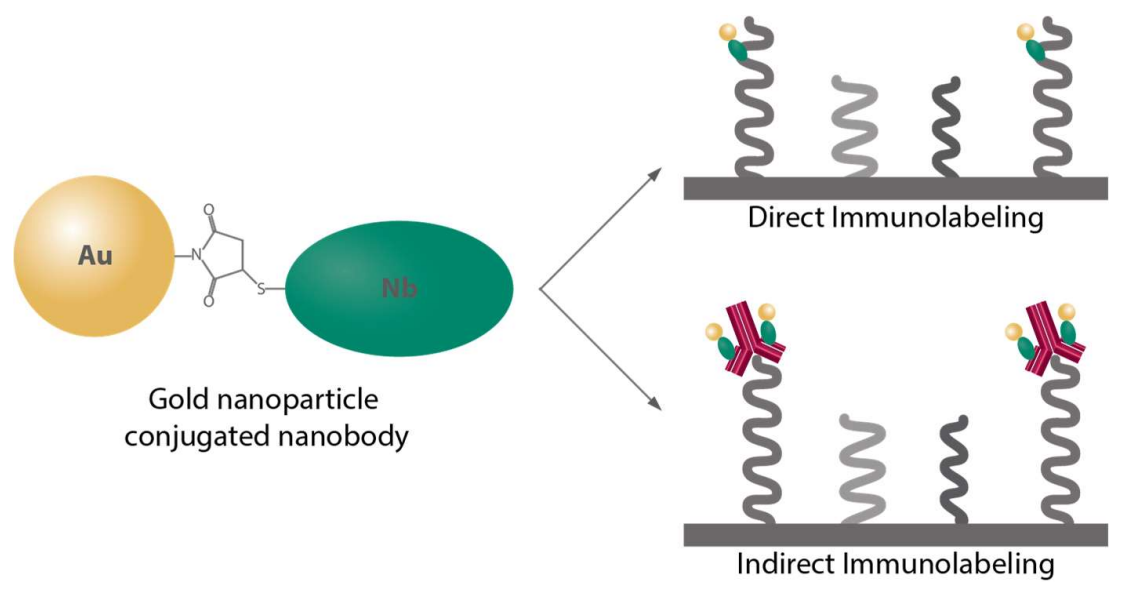

Figure 1. Au nanoprobes for revealing proteins of interest (POIs) via either direct or indirect immunolabeling strategies. Direct immunolabeling is obtained by using an Au nanoprobe detecting endogenous POIs. For indirect immunolabeling, the POIs are first recognized by a specific primary mouse antibody which is then revealed by an Au anti-mouse secondary nanobody.

The Au nanoprobes were tested on different cellular proteins using NanoSIMS imaging. First, the Au anti-vGlut1 nanobody was used to immunostain the vGlut1 in fixed hippocampal neurons from rats. The cells were then embedded in LR White resin, cut into thin sections (200 nm), placed onto silicon wafers, and subsequently measured with NanoSIMS. 
NanoSIMS images were recorded simultaneously for several ions, particularly ${ }^{12} \mathrm{C}^{14} \mathrm{~N},{ }^{28} \mathrm{Si}_{2}$, and ${ }^{197} \mathrm{Au}$. The ion image of ${ }^{197} \mathrm{Au}$ showed that the ${ }^{197} \mathrm{Au}$ signal was considerably higher in the neurite area compared to the cell body (Figure $2 \mathrm{~A}$ ). The ${ }^{12} \mathrm{C}^{14} \mathrm{~N}$ image showed a homogenous ${ }^{12} \mathrm{C}^{14} \mathrm{~N}$ signal across the cell area, and well overlayed with the substrate ${ }^{28} \mathrm{Si}_{2}$, indicating no topographical effect in the sample. The image ratio ${ }^{197} \mathrm{Au} /{ }^{12} \mathrm{C}^{14} \mathrm{~N}$ showed a distinct localization of the nanobody in the neurite area. Particularly, the ${ }^{197} \mathrm{Au}$ signal localizes as "hot spots" along the neurites as expected for the distribution of vGlut in neurons ${ }^{[21,22]}$ (Figure 2C). The line scan profiles across the "hot spot" in the neurites showed significant signal-to-noise of the ${ }^{197} \mathrm{Au}$ signal for the labeled structure (Figure $2 \mathrm{~B}$ and 2D). In addition, the ${ }^{197} \mathrm{Au}$ signal was significantly higher in the cells labeled with the $\mathrm{Au}$ anti-vGlut1 nanobody compared to the non-labeled cells according to the Kolmogorov-Smirnov test at $\mathrm{p}<0.0001$ (Figure $2 \mathrm{~F}$ ). To confirm the localization of the vGlut via the ${ }^{197} \mathrm{Au}$ signal in NanoSIMS, we performed the confocal fluorescence microscopic imaging of vGlut in similar neuronal cells. In this case, the vGlut was labeled with an anti-vGlut1 primary mouse antibody, which was then bound to Abberior STAR 580-anti-mouse secondary antibody. An autofluorescence image of the entire cells was also included for comparing the general shape of the cells with the ${ }^{12} \mathrm{C}^{14} \mathrm{~N}$ image in NanoSIMS. Comparison between the NanoSIMS and confocal images showed a similar distribution of the vGlut in the neurites of the cells (Figure S2). The results showed that Au anti-vGlut1 nanobody was specific for POI, selective, and sufficiently sensitive for detection by NanoSIMS. The imaging resolution of the vGlut1 structure calculated from a line-scan analysis is $\sim 91 \mathrm{~nm}$ (Figure S3).
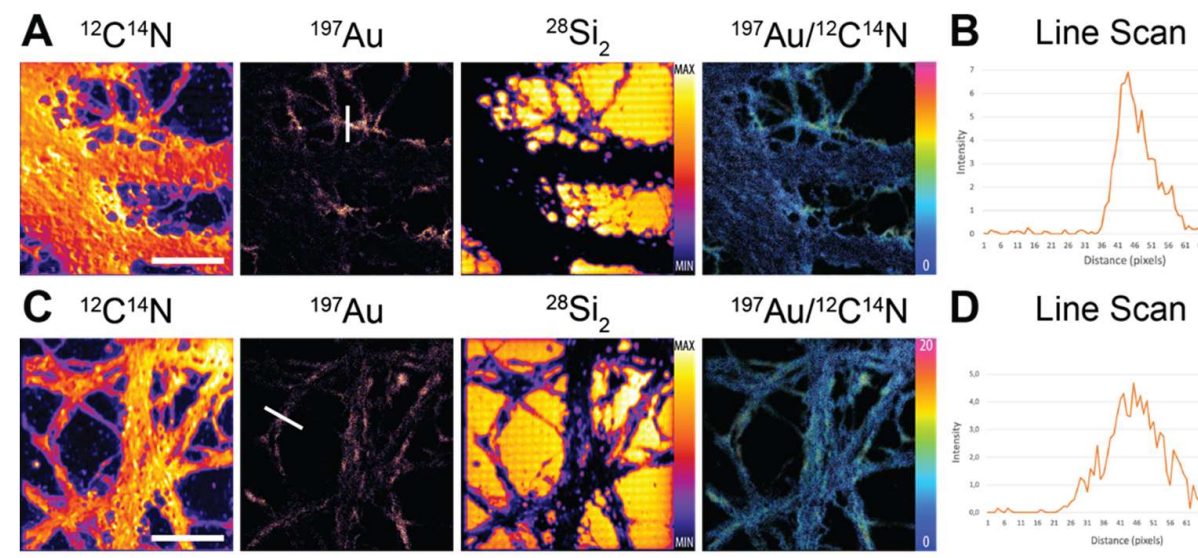

${ }^{197} \mathrm{Au}$
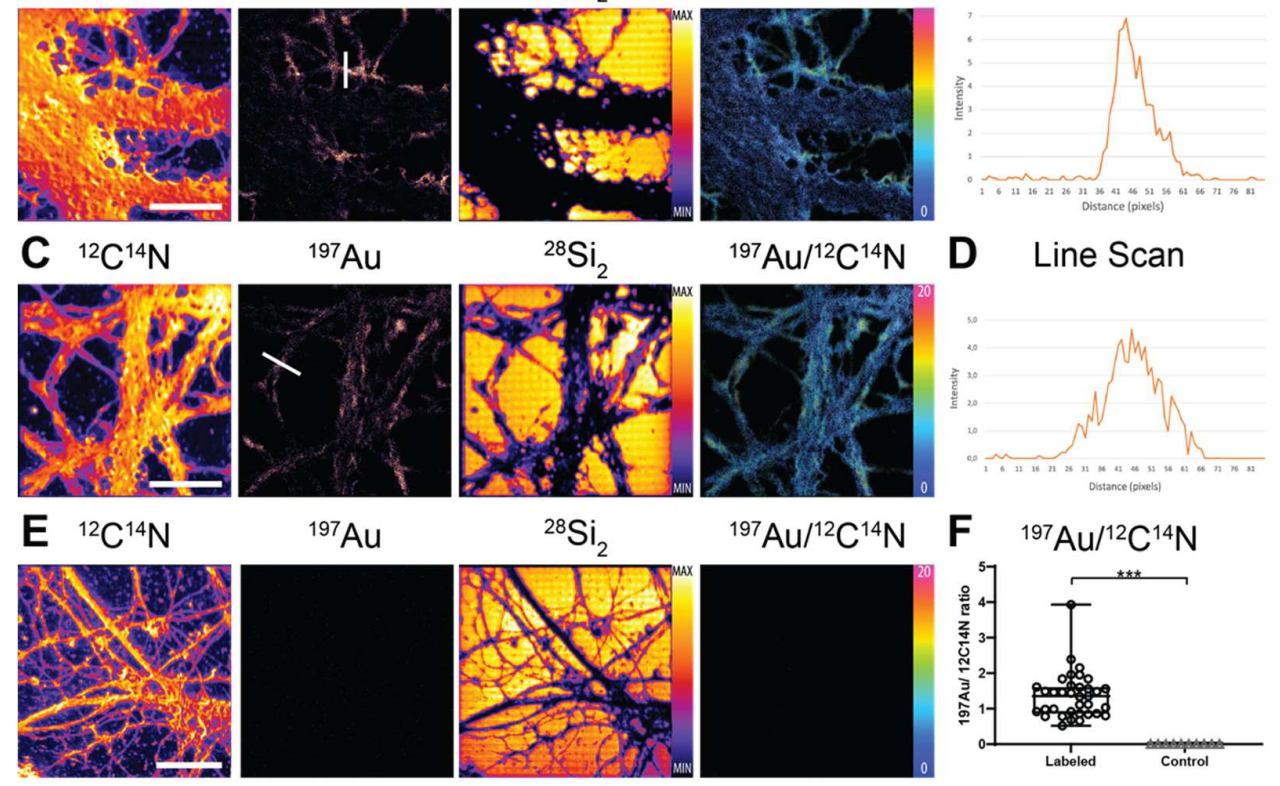

Figure 2. NanoSIMS imaging of vGlut protein in rat hippocampal neurons via direct immunolabeling with the Au anti-vGlut1 nanobody. (A) Cell body and neurites, 512 x 512 pixels. From left to right: ion images of ${ }^{12} \mathrm{C}^{14} \mathrm{~N},{ }^{197} \mathrm{Au},{ }^{28} \mathrm{Si}$, and ${ }^{197} \mathrm{Au} /{ }^{12} \mathrm{C}^{14} \mathrm{~N}$ ratio. (B) A line-scan profile of ${ }^{197} \mathrm{Au}$ signal across a neurite in ${ }^{197} \mathrm{Au}$ image (white line). (C) Neurites, 512 x 512 pixels. From left to right: ion images of ${ }^{12} \mathrm{C}^{14} \mathrm{~N},{ }^{197} \mathrm{Au},{ }^{28} \mathrm{Si}$, and ${ }^{197} \mathrm{Au} /{ }^{12} \mathrm{C}^{14} \mathrm{~N}$ ratio. (D) A line-scan profile of ${ }^{197} \mathrm{Au}$ signal across a neurite in ${ }^{197} \mathrm{Au}$ image (white line). (E) Negative control cell without Au anti-vGlut1 nanobody. From left to right: ion images of ${ }^{12} \mathrm{C}^{14} \mathrm{~N},{ }^{197} \mathrm{Au}$, ratio ${ }^{197} \mathrm{Au} /{ }^{12} \mathrm{C}^{14} \mathrm{~N}$, and ${ }^{28} \mathrm{Si}_{2}$. (F) Comparison of signal intensity of ${ }^{197} \mathrm{Au}$ between the cells labeled with the Au anti-vGlut1 nanobody and the negative control cells. Significantly higher signal in the labeled cells compared to the control cells verified by a Kolmogorov-Smirnov test $(\mathrm{p}<0.0001)(\mathrm{n}=35$ for labeled cells, $\mathrm{n}=10$ for control cells). Error bars show the SEM. Scale bars are $10 \mu \mathrm{m}$. 
We then tested the second type of nanoprobe, Au anti-mouse secondary nanobody, for different proteins, including the synaptic protein Synaptotagmin-1 and the mitochondrial marker TOM20. The fixed neurons were first immunoassayed with a mouse primary antibody that recognized the POIs followed by the Au anti-mouse secondary nanobody. The labeled cells were then let air-dried before the NanoSIMS measurement.

The ${ }^{12} \mathrm{C}^{14} \mathrm{~N}$ signal was not evenly distributed across the NanoSIMS images, which could account for a low degree of sample topography (Figure 3) due to the use of the whole dried cells for imaging. However, the use of the signal ratio of ${ }^{197} \mathrm{Au} /{ }^{12} \mathrm{C}^{14} \mathrm{~N}$, in this case, could help reduce the difference in the secondary ion yield caused by the topographical effect. For all the samples, the signal of ${ }^{12} \mathrm{C}^{14} \mathrm{~N}$ was overlayed well with the ${ }^{28} \mathrm{Si}$. For the Synaptotagmin-1 labeling, the ${ }^{197} \mathrm{Au}$ signal localized as small speckles along the neurites which is in good agreement with other studies ${ }^{[23,24]}$ (Figure 3A). The labeling of mitochondrial marker TOM20 showed the distribution in the cytoplasm across the cells [25] (Figure 3B). The distribution of TOM20 in the NanoSIMS images appeared with a similar pattern to that observed in the confocal fluorescence images of TOM20 revealed by Cy5-antimouse secondary antibody (Figure S4). In the negative control cells labeled with the Au anti-mouse secondary nanobody in the absence of the primary antibody, there was a low level of ${ }^{197} \mathrm{Au}$ signal, which is possibly caused by a small extent of non-specific binding of the nanobody (Figure 3C). However, the ${ }^{197} \mathrm{Au}$ signal in the control samples was significantly lower compared to that in the labeled cells. Furthermore, a negligible signal of ${ }^{197} \mathrm{Au}$ was detected in the negative control cells labeled with neither the primary antibody nor the Au anti-mouse secondary nanobody (Figure 3D). The labeled cells showed a statistically higher ${ }^{197} \mathrm{Au}$ signal compared to those of the negative control cells based on the Kolmogorov-Smirnov test $(\mathrm{p}<0.0001)$ (Figure S5). The Au anti-mouse secondary nanobody was shown its sufficient selectivity to the corresponding primary antibody, high flexibility for the two examined target proteins, and sufficient sensitivity for the NanoSIMS measurement. The imaging resolution of the structures of TOM20 and synaptotagmin1 calculated from a line-scan analysis are approximately $116 \mathrm{~nm}$ and $981 \mathrm{~nm}$ (Figure S3). Synaptotagmin1 has a worse resolution compared to TOM20 because a bigger diaphragm D1-1 was used to increase the detected signal for synaptotagmin1. 

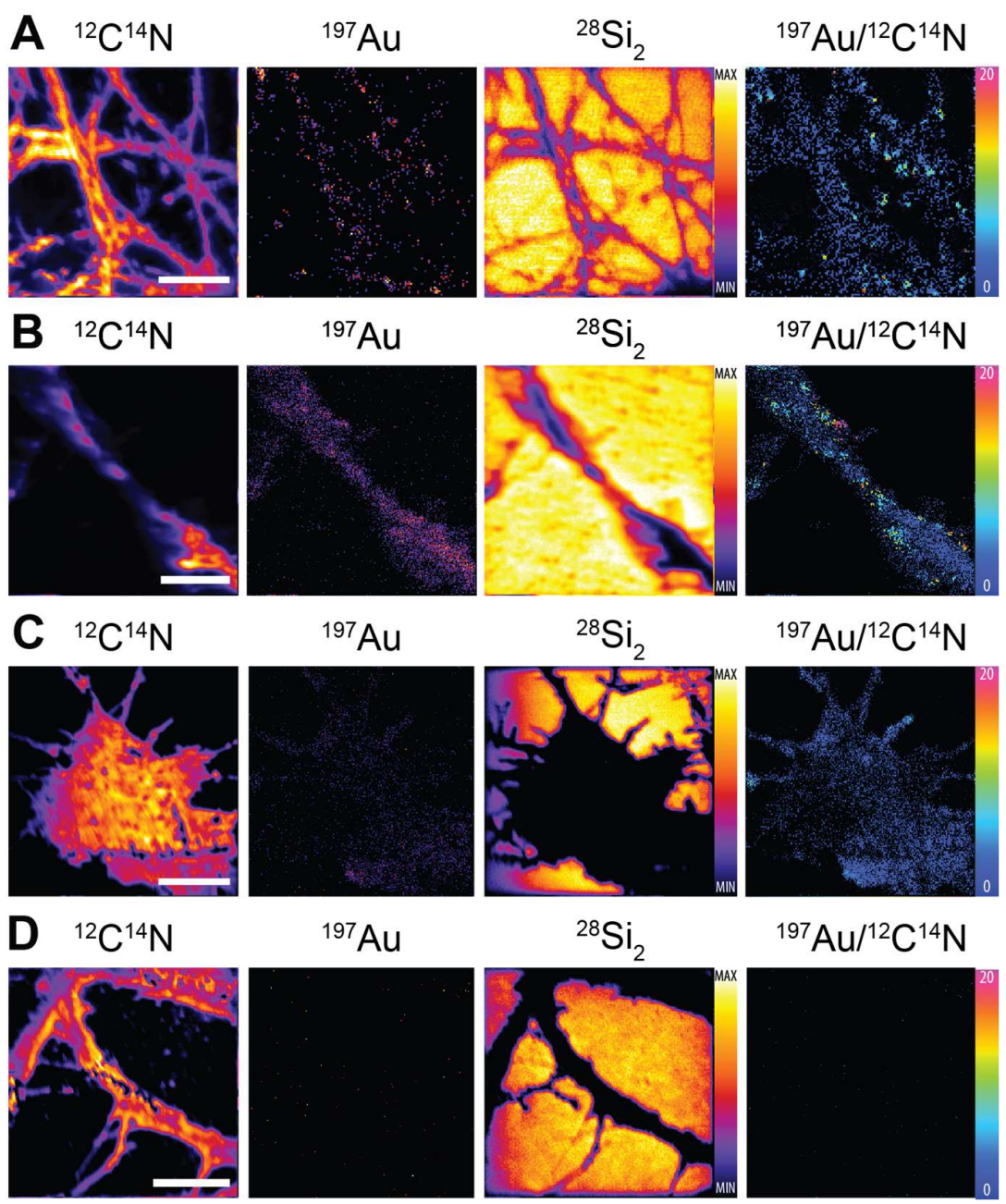

${ }^{197} \mathrm{Au} /{ }^{12} \mathrm{C}^{14} \mathrm{~N}$

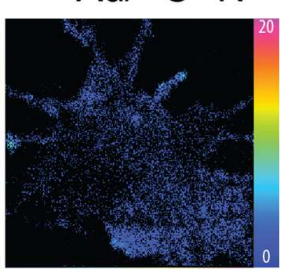

Figure 3. NanoSIMS imaging of specific proteins in hippocampal neurons via indirect immunolabeling with the Au anti-mouse secondary nanobody. (A) Synaptic protein Synaptotagmin1. 128 x 128 pixels. From left to right: ion images of ${ }^{12} \mathrm{C}^{14} \mathrm{~N},{ }^{197} \mathrm{Au},{ }^{28} \mathrm{Si}$, and ${ }^{197} \mathrm{Au} /{ }^{12} \mathrm{C}^{14} \mathrm{~N}$ ratio. (B) Mitochondrial marker TOM20. $256 \times 256$ pixels. From left to right: ion images of ${ }^{12} \mathrm{C}^{14} \mathrm{~N},{ }^{197} \mathrm{Au},{ }^{28} \mathrm{Si}$, and ${ }^{197} \mathrm{Au} /{ }^{12} \mathrm{C}^{14} \mathrm{~N}$ ratio. (C) Negative control cell with $\mathrm{Au}$ anti-mouse secondary nanobody in the absence of primary antibody. $256 \times 256$ pixels. From left to right: ion images of ${ }^{12} \mathrm{C}^{14} \mathrm{~N},{ }^{197} \mathrm{Au},{ }^{28} \mathrm{Si}$, and ${ }^{197} \mathrm{Au} /{ }^{12} \mathrm{C}^{14} \mathrm{~N}$ ratio. (D) Negative control cell in the absence of both primary antibody and Au anti-mouse secondary nanobody. From left to right: ion images of ${ }^{12} \mathrm{C}^{14} \mathrm{~N},{ }^{197} \mathrm{Au},{ }^{28} \mathrm{Si}_{2}$, and ${ }^{197} \mathrm{Au} /{ }^{12} \mathrm{C}^{14} \mathrm{~N}$ ratio. Scale bars for (A), (C), and (D) are $10 \mu \mathrm{m}$. The scale bar for (B) is $3 \mu \mathrm{m}$.

\section{Discussion}

We have previously developed several dual-labeling probes for the detection of specific cellular proteins. Each probe had a fluorophore and an isotopic element, such as bo$\operatorname{ron}^{[4]}$ or fluorine ${ }^{[5]}$, allowing the imaging with both fluorescence microscopy and NanoSIMS on the same sample. These probes were shown high performance in terms of imaging resolution, labeling precision, and labeling density. However, they could encounter a problem with the high background signal from the embedding resin and the substrate materials. In this work, we have generated the gold anti-mouse secondary nanobody and have demonstrated its applications, in parallel with a custom-made product $\mathrm{Au}$ antivGlut1 nanobody, for immunolabeling various cellular proteins and imaging with $\mathrm{Na}-$ noSIMS. The Au NPs provide a high signal to background in SIMS as they are rarely present in biological and embedding materials. Immuno-detection is a straightforward approach, allowing the nanoprobes to reveal the endogenous proteins without any genetic manipulation. There are advantages and disadvantages of using conjugated nanobody or 
conjugated antibody. While the nanobody can penetrate better to its epitopes due to its small size compared to antibody, such Au particles linked to the antibody ${ }^{[10]}$ would result in a higher amount of $\mathrm{Au}$ atoms per epitope, thus higher sensitivity for SIMS measurement, compared to the nanobody, since multiple particles are linked to each antibody. However, the conjugation of Au particles to the antibody is less reproducible than the procedure for nanobody. The reason is that the nanobody has comparatively few amino acid side chains, it can be linked to the gold particles at specific sites, thereby ensuring that every single batch of nanobody is conjugated in the same fashion.[26] On the other hand, antibody is much larger and has more amino acid side chains, its conjugation to various labels is difficult to standardize. For example, using amine-reactive Au particles ${ }^{[10]}$ would link the Au particles to different site chains on each antibody leading to a large variability from batch to batch of the conjugation.

The Au anti-vGlut1 nanobody allows a direct binding of the POI vGlut, which offers a high specificity, high labeling precision, and imaging at nanoscale resolution. The direct labeling nanoprobe, however, cannot be flexibly used for many proteins, but different proteins require different Au coupled nanobodies to specifically recognize their epitopes. In addition, the palette of available nanobodies recognizing endogenous proteins is currently very limited. This limitation can be alleviated by the Au anti-mouse secondary nanobody, which enables a flexible application of the nanoprobe to any POI that binds to their mouse antibodies. From our results, the indirect labeling nanoprobe was shown to exhibit high selectivity and sensitivity for target molecular imaging with NanoSIMS. Moreover, this type of probe also allows the amplification in which multiple steps of labeling using different primary antibodies are performed in order to increase the detectability of low abundant proteins on NanoSIMS. However, this could affect the labeling precision because the ${ }^{197} \mathrm{Au}$ signal is placed far away from the epitopes. Further development of the nanoprobes is highly desirable to increase the sensitivity for SIMS detection by increasing the labeling sites of Au NPs on each nanobody alternatively to the multiple labeling approach. However, this needs a careful design to avoid the risk of precipitation caused by an increased amount of Au NPs per nanobody.

The gold nanoprobes are also applicable to other SIMS techniques such as ToF-SIMS, allowing the detection of various molecules, including metabolites, lipids, and small peptides in relation to the localization of specific cellular structures labeled with the Au nanoprobes. As ToF-SIMS is well known as a label-free technique, labeling has not been commonly applied in this field. However, ToF-SIMS has a limited $\mathrm{m} / \mathrm{z}$ range of detection in which large molecules such as proteins cannot be analyzed. This can be complemented by employing labeling probes for specific peptides and proteins [27-29]. For example, one application of antibody in ToF-SIMS imaging is the use of antibody coupled liposomes for simultaneous detection of amyloid- $\beta$, a main component of plaques in Alzheimer's disease (AD), and lipids in the AD mouse brain ${ }^{[27]}$. Another example is the multiplexed dynamic ToF-SIMS imaging for characterization of different tumor microenvironments in tumors using 15 antibodies, each coupled with specific metal isotopes [29]. These studies demonstrate the value of implementing the information in ToF-SIMS to study a complex interaction of different molecules in diseases such as neurodegeneration and cancer. Nevertheless, these antibodies could only be used for labeling specific proteins (direct labeling approach). In our case, the Au anti-mouse secondary nanobody provides a flexibility in labeling different proteins of interest. The monovalent nature and small size of the nanobody used for the nanoprobes eliminate the risk of clustering. In addition, the Au element can be detected with higher sensitivity by ToF-SIMS compared to other probes containing light elements such as boron or fluorine ${ }^{[4-6]}$.

The gold-nanoprobes would enable the correlation of NanoSIMS and EM imaging to obtain multidimensional information of the samples, which cannot be obtained by the individual technique, particularly the turnover of the isotopic molecules and the localization of the protein architecture on NanoSIMS and its morphological property on EM.

\section{Conclusions}


We have successfully applied the Au nanoprobes, which contain Au NPs conjugated to nanobodies, to image different target proteins in hippocampal neurons using NanoSIMS. The nanoprobes were shown well suited for SIMS imaging at subcellular resolution with sufficient sensitivity and high specificity to the proteins of interest. The direct and indirect labeling nanoprobes enable flexible immunostaining for a broad range of proteins to be imaged by NanoSIMS. The nanoprobes can also be used for other SIMS techniques allowing simultaneous imaging of specific cellular proteins and various analytes, including small molecules, metabolites, and lipids. Furthermore, the Au nanoprobes offer the possibility to correlate NanoSIMS and EM imaging to understand the functional and structural relationship at the subcellular level by combining the information of the cellular turnover, protein localization and cell morphology.

Supplementary Materials. Size-exclusion chromatograph of gold and anti-mouse nanobody conjugation. Laser scanning confocal fluorescence images of vGlut in hippocampal neurons, confocal fluorescence images of mitochondrial marker TOM20 in hippocampal neurons, line-scans for determining spatial resolution for vGlut1, TOM20, and synaptotagmin1, and a chart compares ${ }^{197} \mathrm{Au}$ signal in the neuronal cells labeled with TOM20 plus Au anti-mouse secondary nanobody compared to the negative control cells.

Author Contributions. Conceptualization, Silvio O. Rizzoli and Nhu T. N. Phan; Formal analysis, Paola Agüi-Gonzalez and Nhu T. N. Phan; Funding acquisition, Silvio O. Rizzoli and Nhu T. N. Phan; Methodology, Paola Agüi-Gonzalez, Tal Dankovich, Felipe Opazo, Silvio O. Rizzoli and Nhu T. N. Phan; Supervision, Silvio O. Rizzoli and Nhu T. N. Phan; Validation, Paola Agüi-Gonzalez, Silvio O. Rizzoli and Nhu T. N. Phan; Writing - original draft, Paola Agüi-Gonzalez and Nhu T. N. Phan; Writing - review \& editing, Paola AgüiGonzalez, Tal Dankovich, Felipe Opazo, Silvio O. Rizzoli and Nhu T. N. Phan.

Funding. This research was funded by the Deutsche Forschungsgemeinschaft (DFG, SFB 1286/B1), VR (Swedish Research Council, 2016-06800), and the Hasselblad Foundation to to N.T.N.P, and by the DFG (SFB 1286/A3) to S.O.R.

Data Availability Statement. The data presented in this study are available on request to the corresponding author.

Conflicts of Interest. F.O and S.O.R, are shareholders of NanoTag Biotechnologies GmbH

\section{References}

1. Saka, S. K.; Vogts, A.; Kröhnert, K.; Hillion, F.; Rizzoli, S. O.; Wessels, J. T. Correlated Optical and Isotopic Nanoscopy. Nat. Commun. 2014, 5 (1), 3664. https://doi.org/10.1038/ncomms4664.

2. Lovrić, J.; Dunevall, J.; Larsson, A.; Ren, L.; Andersson, S.; Meibom, A.; Malmberg, P.; Kurczy, M. E.; Ewing, A. G. Nano Secondary Ion Mass Spectrometry Imaging of Dopamine Distribution Across Nanometer Vesicles. ACS Nano 2017, 11 (4), 3446-3455. https://doi.org/10.1021/acsnano.6b07233.

3. Eijkel, G. B.; Kükrer Kaletaş, B.; van der Wiel, I. M.; Kros, J. M.; Luider, T. M.; Heeren, R. M. A. Correlating MALDI and SIMS Imaging Mass Spectrometric Datasets of Biological Tissue Surfaces. Surf. Interface Anal. 2009, 41 (8), 675-685. https://doi.org/10.1002/sia.3088.

4. Kabatas, S.; Agüi-Gonzalez, P.; Saal, K. A.; Jähne, S.; Opazo, F.; Rizzoli, S. O.; Phan, N. T. N. Boron-Containing Probes for NonOptical High-Resolution Imaging of Biological Samples. Angew. Chemie - Int. Ed. 2019, 58 (11), 3438-3443. https://doi.org/10.1002/anie.201812032.

5. Kabatas, S.; Agüi-Gonzalez, P.; Hinrichs, R.; Jähne, S.; Opazo, F.; Diederichsen, U.; Rizzoli, S. O.; Phan, N. T. N. Fluorinated Nanobodies for Targeted Molecular Imaging of Biological Samples Using Nanoscale Secondary Ion Mass Spectrometry. J. Anal. At. 
Spectrom. 2019, 34 (6), 1083-1087. https://doi.org/10.1039/C9JA00117D.

6. Vreja, I. C.; Kabatas, S.; Saka, S. K.; Kröhnert, K.; Höschen, C.; Opazo, F.; Diederichsen, U.; Rizzoli, S. O. Secondary-Ion Mass Spectrometry of Genetically Encoded Targets. Angew. Chemie Int. Ed. 2015, 54 (19), 5784-5788.

https://doi.org/10.1002/anie.201411692.

7. Wilson, R. L.; Frisz, J. F.; Hanafin, W. P.; Carpenter, K. J.; Hutcheon, I. D.; Weber, P. K.; Kraft, M. L. Fluorinated Colloidal Gold Immunolabels for Imaging Select Proteins in Parallel with Lipids Using High-Resolution Secondary Ion Mass Spectrometry. Bioconjug. Chem. 2012, 23 (3), 450-460. https://doi.org/10.1021/bc200482z.

8. Lou, X.; Zhang, G.; Herrera, I.; Kinach, R.; Ornatsky, O.; Baranov, V.; Nitz, M.; Winnik, M. A. Polymer-Based Elemental Tags for Sensitive Bioassays. Angew. Chemie Int. Ed. 2007, 46 (32), 6111-6114. https://doi.org/10.1002/anie.200700796.

9. Adams, S. R.; Mackey, M. R.; Ramachandra, R.; Palida Lemieux, S. F.; Steinbach, P.; Bushong, E. A.; Butko, M. T.; Giepmans, B. N. G.; Ellisman, M. H.; Tsien, R. Y. Multicolor Electron Microscopy for Simultaneous Visualization of Multiple Molecular Species. Cell Chem. Biol. 2016, 23 (11), 1417-1427. https://doi.org/10.1016/j.chembiol.2016.10.006.

10. Thiery-Lavenant, G.; Guillermier, C.; Wang, M.; Lechene, C. Detection of Immunolabels with Multi-Isotope Imaging Mass Spectrometry. Surf. Interface Anal. 2014, 46 (S1), 147-149. https://doi.org/10.1002/sia.5596.

11. Micheva, K. D.; Smith, S. J. Array Tomography: A New Tool for Imaging the Molecular Architecture and Ultrastructure of Neural Circuits. Neuron 2007, 55 (1), 25-36. https://doi.org/10.1016/j.neuron.2007.06.014.

12. Holderith, N.; Heredi, J.; Kis, V.; Nusser, Z. A High-Resolution Method for Quantitative Molecular Analysis of Functionally Characterized Individual Synapses. Cell Rep. 2020, 32 (4), 107968. https://doi.org/10.1016/j.celrep.2020.107968.

13. Angelo, M.; Bendall, S. C.; Finck, R.; Hale, M. B.; Hitzman, C.; Borowsky, A. D.; Levenson, R. M.; Lowe, J. B.; Liu, S. D.; Zhao, S.; Natkunam, Y.; Nolan, G. P. Multiplexed Ion Beam Imaging of Human Breast Tumors. Nat. Med. 2014, 20 (4), 436-442. https://doi.org/10.1038/nm.3488.

14. Sograte-Idrissi, S.; Schlichthaerle, T.; Duque-Afonso, C. J.; Alevra, M.; Strauss, S.; Moser, T.; Jungmann, R.; Rizzoli, S. O.; Opazo, F. Circumvention of Common Labelling Artefacts Using Secondary Nanobodies. Nanoscale 2020, 12 (18), 10226-10239. https://doi.org/10.1039/d0nr00227e.

15. Pilolli,R; Palmisano, F; Cioffi, Ni.Gold nanomaterials as a new tool for bioanalytical applications of laser desorption ionization mass spectrometry. Anal Bioanal Chem 2012, 402, 601-623. https://doi.org/10.1007/s00216-011-5120-2.

16. Zhu, Z-J; Rotello, V. M.; Vachet, R. W. Engineered nanoparticle surfaces for improved mass spectrometric analyses. Analyst 2009, 134, 2183-2188. https://doi.org/10.1039/b910428c.

17. Amoroso, A. J. and Pope, S. J. A. Using lanthanide ions in molecular bioimaging. Chem. Soc. Rev. 2015, 44, 4723. https://doi.org/10.1039/c4cs00293h.

18. Lang, P. F. and Smith, B. C. Ionization Energies of Lanthanides. J. Chem. Educ. 2010, 87, 8, 875-881. https://doi.org/10.1021/ed100215q.

19. Andersen, T., Haugen, H. L; and Hotop, H. Binding Energies in Atomic Negative Ions: III. Journal of Physical and Chemical Reference Data 1999, 28(6), 1511-33. https://doi.org/10.1063/1.556047.

20. Rueden, C. T.; Schindelin, J.; Hiner, M. C.; DeZonia, B. E.; Walter, A. E.; Arena, E. T.; Eliceiri, K. W. ImageJ2: ImageJ for the next Generation of Scientific Image Data. BMC Bioinformatics 2017, 18 (1), 529. https://doi.org/10.1186/s12859-017-1934-z.

21. Wojcik, S. M.; Rhee, J. S.; Herzog, E.; Sigler, A.; Jahn, R.; Takamori, S.; Brose, N.; Rosenmund, C. An Essential Role for Vesicular Glutamate Transporter 1 (VGLUT1) in Postnatal Development and Control of Quantal Size. Proc. Natl. Acad. Sci. U. S. A. 2004, 101 (18), 7158-7163. https://doi.org/10.1073/pnas.0401764101.

22. Bhargava, N.; Das, M.; Edwards, D.; Stancescu, M.; Kang, J. F.; Hickman, J. J. Coexpression of Glutamate Vesicular Transporter (VGLUT1) and Choline Acetyltransferase (ChAT) Proteins in Fetal Rat Hippocampal Neurons in Culture. Vitr. Cell. Dev. Biol. - Anim. 2010, 46 (8), 685-692. https://doi.org/10.1007/s11626-010-9324-0.

23. Inoue, Y.; Kamikubo, Y.; Ezure, H.; Ito, J.; Kato, Y.; Moriyama, H.; Otsuka, N. Presynaptic Protein Synaptotagmin1 Regulates the Neuronal Polarity and Axon Differentiation in Cultured Hippocampal Neurons. BMC Neurosci. 2015, 16 (1). 
https://doi.org/10.1186/s12868-015-0231-x.

24. Jia, J. Y.; Lamer, S.; Schümann, M.; Schmidt, M. R.; Krause, E.; Haucke, V. Quantitative Proteomics Analysis of Detergent-Resistant Membranes from Chemical Synapses: Evidence for Cholesterol as Spatial Organizer of Synaptic Vesicle Cycling. Mol. Cell. Proteomics 2006, 5 (11), 2060-2071. https://doi.org/10.1074/mcp.M600161-MCP200.

25. Amadoro, G.; Corsetti, V.; Florenzano, F.; Atlante, A.; Bobba, A.; Nicolin, V.; Nori, S. L.; Calissano, P. Morphological and Bioenergetic Demands Underlying the Mitophagy in Post-Mitotic Neurons: The Pink-Parkin Pathway. Front. Aging Neurosci. $2014,6$. https://doi.org/10.3389/fnagi.2014.00018.

26. Pleiner, T; Bates, M; Trakhanov, S; Lee, CT; Schliep, JE; Chug, H; Böhning, M; Stark, H; Urlaub, H; Görlich, D. Nanobodies: sitespecific labeling for super-resolution imaging, rapid epitope-mapping and native protein complex isolation. Elife. 2015, 3(4), e11349. https://doi.org/10.7554/eLife.11349.

27. Carlred, L.; Gunnarsson, A.; Solé-Domènech, S.; Johansson, B.; Vukojević, V.; Terenius, L.; Codita, A.; Winblad, B.; Schalling, M.; Höök, F.; Sjövall, P. Simultaneous Imaging of Amyloid- $\beta$ and Lipids in Brain Tissue Using Antibody-Coupled Liposomes and Timeof-Flight Secondary Ion Mass Spectrometry. J. Am. Chem. Soc. 2014, 136 (28), 9973-9981. https://doi.org/10.1021/ja5019145.

28. Gularyan, S. K.; Gulin, A. A.; Anufrieva, K. S.; Shender, V. O.; Shakhparonov, M. I.; Bastola, S.; Antipova, N. V.; Kovalenko, T. F.; Rubtsov, Y. P.; Latyshev, Y. A.; Potapov, A. A.; Pavlyukov, M. S. Investigation of Inter- And Intratumoral Heterogeneity of Glioblastoma Using TOF-SIMS. Mol. Cell. Proteomics 2020, 19 (6), 960-970. https://doi.org/10.1074/mcp.RA120.001986.

29. Ptacek, J.; Locke, D.; Finck, R.; Cvijic, M. E.; Li, Z.; Tarolli, J. G.; Aksoy, M.; Sigal, Y.; Zhang, Y.; Newgren, M.; Finn, J. Multiplexed Ion Beam Imaging (MIBI) for Characterization of the Tumor Microenvironment across Tumor Types. Lab. Investig. 2020, 100 (8), 1111-1123. https://doi.org/10.1038/s41374-020-0417-4. 